\title{
GEORREFERENCIAMENTO DE CASOS DE LEISHMANIOSE VISCERAL NA CIDADE DE TEÓFILO OTONI-MGDO ANO DE 2008 A 2017
}

\section{GEORREFERENCING LEISHMANIASIS VISCERAL CASES IN THE CITY OF TEOFILO OTONI-MG FROM 2008 TO 2017}

\author{
Issamara Oliveira Fonseca \\ Acadêmica do curso de farmácia $9^{\circ}$ período da Faculdade Presidente Antônio Carlos \\ UNIPAC Teófilo Otoni-MG email: issa_mara2011@hotmail.com \\ Thamires Macedo Nunes \\ Acadêmica do curso de farmácia $9^{\circ}$ período da Faculdade Presidente Antônio Carlos \\ UNIPAC Teófilo Otoni-MG - email: thamiresmanu22@gmail.com \\ Médico Veterinário. Professor da Faculdade Presidente Antônio Carlos UNIPAC Teófilo \\ Otoni-MG \\ Orientador - email: lucioonofri@gmail.com
}

Daniel de Azevedo Teixeira Farmacêutico bioquímico, Professor da Faculdade Presidente Antônio Carlos de Teófilo Otoni-MG email: daneil@gmail.com

\section{Resumo}

A cidade de Teófilo Otoni apresenta clima e características propícios para a proliferação do vetor da leishmaniose visceral, que se reproduz em locais úmidos e com material orgânico em decomposição, tornando difícil o seu controle. As medidas profiláticas da leishmaniose são aplicadas em todo território da cidade, no entanto acredita-se que uma intervenção específica nos focos principais apresentariam melhores resultados no controle de reprodução do vetor. A pesquisa teve como objetivo realizar um levantamento das áreas com maior registro de casos no município, caracterizando os bairros mais acometidos e mensurar o risco de uma epidemia da doença na cidade. Através do sistema de informação geográfica (SIG), foi feito o mapeamento das áreas mais acometidas pela leishmaniose para definir os pontos críticos de surgimento de novos casos. Foi realizada uma coleta de dados junto ao SINAN e na Superintendência Municipal de Saúde de Teófilo Otoni avaliando-se variáveis como: incidência por bairro, ocorrência por gênero, faixa etária dos pacientes notificados e condições socioeconômicas da região.Foram confirmados 19 casos na cidade no período de estudo. A população masculina corresponde a $79 \%$ desses casos enquanto a feminina representa $21 \%$. Houve grande prevalência de $57,90 \%$ na população infantil e 42,1 na população adulto e senil juntas. Os bairros mais incidentes foram: São Cristóvão (4 casos), Bela Vista (4 casos) e Joaquim Pedrosa (2 casos). Foi constatado que a maior concentração de 
confirmações foi na região noroeste da cidade e que está relacionado com os aspectos ambientais e socioeconômicos da região.

Palavras-Chave: Georreferenciamento de doenças; Leishmaniose Visceral; Epidemiologia; Vigilância Epidemiológica; Saúde Pública.

\section{Abstract}

Teófilo Otoni city presents a climate and characteristics favorable for the proliferation of the transmitter of visceral leishmaniasis, which reproduces in humid places and decomposing organic material, making its controlvery difficult. Prophylactic means against leishmaniasis are applied throughout the territory of the city, however it is believed that a specific intervention in the main foci would present better results in the vector reproduction control. The aim of this research was to survey areas with the highest number of cases in the city, characterizing the most affected neighborhoods and measuring the risk of an outbreak of the disease in the city. Through the geographic information system (GIS), the mapping of the areas most affected by leishmaniasis was done to define the critical points for new cases to appear. Data were collected from SINAN and the Municipal Health Headquarter of Teófilo Otoni, evaluating variables such as: incidence by neighborhood, occurrence by gender, age of notified patients, and socioeconomic conditions of the region. We confirmed 19 cases of LV in the city during the study period. The male population corresponds to $79 \%$ of these cases while the female population represents $21 \%$. There was a high prevalence of $57.90 \%$ in the infant population and 42.1 in the adult and senile population together. The most incident neighborhoods were São Cristovão (4 cases), Bela Vista (4 cases) and Joaquim Pedrosa (2 cases). It was found that the highest concentration of LV confirmations was in the northwest of the city and that it is related to the environmental and socioeconomic aspects of the region.

Keywords: Georreferencing of diseases; Visceral Leishmaniasis; Epidemiology; Epidemiological surveillance; Public health.

\section{Introdução}

A Leishmaniose Visceral (LV) também conhecida por calazar é uma doença parasitária infecciosa, distribuída em todo mundo, que afeta o homem e animais. A taxa de mortalidade associada a essa doença a tornou caso de saúde pública em pelo menos 88 países (ALVARENGA et al., 2010). Em Bangladesh, Sudão, Índia, Brasil e Nepal ocorreram 90\% dos casos registrados no mundo (MURRAY, 2004).

Segundo a Organização Mundial da Saúde (OMS), a LV é considerada de caráter prioritário, devendo todos os casos serem registrados pela notificação compulsória (OLIVEIRA, 2011). 
Causada pelos protozoários dos gêneros Leishmania donovani, Leishmania chagasi e Leishmania infantum, a doença é transmitida pelo flebotomíneo fêmea das espécies Lutzomyia longipalpis e Lutzomyia cruzinnfectados e possui uma gama considerável de manifestações clínicas, que varia de acordo com a espécie deLeishmania implicada (MICHALICK; GENARO, 2005)

FIGURA 1. Ilustração do flebotomíneo do gênero Lutzomyia spp.

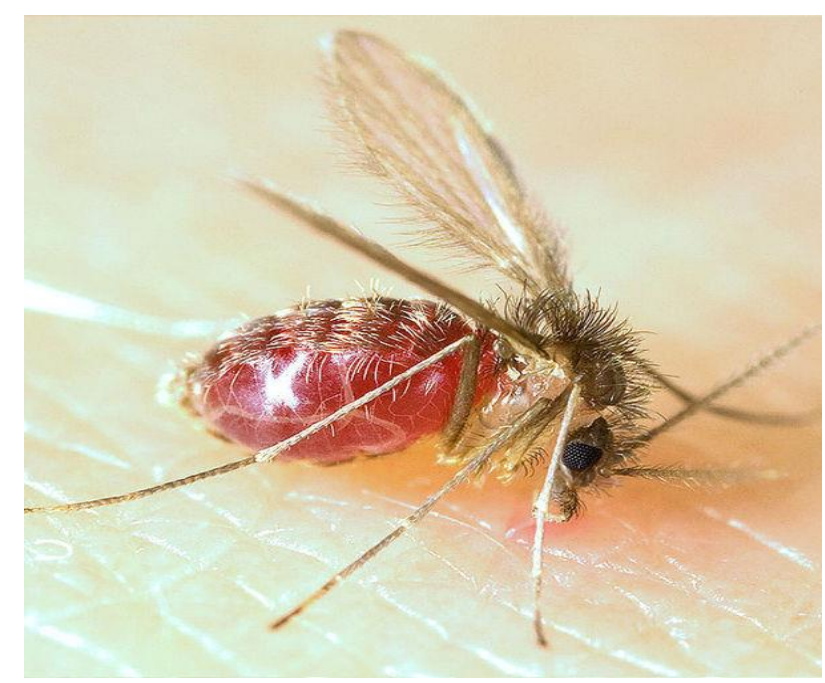

Fonte: Wilson (2009)

Os quadros clínicos da doença estão associados a febre intermitente, palidez, emagrecimento progressivo, hepatoesplenomegalia e fraqueza generalizada.O diagnóstico da LV é feito inicialmente pela pesquisa do parasito em amostras biológicas de tecidos do paciente. Outros métodos sorológico como o Ensaio Imunoenzimático ligado a enzimas (ELISA) ou Reação de Imunofluorescência (RIFI) também podem ser solicitados para confirmação dos resultados. A terapêutica medicamentosa para tratamento da LV é limitada. As drogas utilizadas são os antimoniais pentavalentes - antimoniato de $\mathrm{N}$ metilglucamina (Glucantime®) e o estiobogliconato sódico (Pentostam). O Glucantime ${ }^{\circledR}$ é a primeira opção para o tratamento dessa patologia no Brasil. A finalização no tratamento é fundamental para a redução da resistência do parasito ao medicamento (MICHALICK; GENARO, 2005).

Anteriormente considerada de natureza rural, a LV é frequentemente registrada na zona urbana e os cães são considerados os principais reservatórios do protozoário na referida localidade. No entanto, a OMS destaca que uma das maiores 
dificuldades no combate ao vetor da LV é o fato de inúmeros animais atuarem como reservatório do parasita (WHO, 1990)

As medidas de controle da LV se baseiam no tratamento dos indivíduos positivos, controle dos reservatórios, manejo ambiental e atuação constante da vigilância epidemiológica (BRASIL, 2006).Barata et al.(2011) dizem que a eficácia desses métodos depende dos aspectos epidemiológicos de cada região.

No Brasil a média de incidência anual é de aproximadamente 3.156 casos e o Ministério da saúde apontou o estado de Minas Gerais com o maior número de registros da doença no ano de 2015(BRASIL, 2006).

Um boletim epidemiológico lançado pela Secretaria de Estado de Saúde de Minas Gerais mostra que no período de 2010 a 2015, 7788 casos novos de LV foram notificados no Estado, dos quais 2456 foram confirmados.Dos casos confirmados, Belo Horizonte apresenta o maior número com $36,9 \%$ dos casos e Teófilo Otoni representa $0,9 \%$.(MINAS GERAIS, 2017). Esse número de casos em tão pouco tempo, coloca a cidade de Teófilo Otoni em alerta quanto à necessidade da amplificação de medidas de controle da doença na região.

Esta elevação global dos casos de LV observados nas zonas endêmicas nos últimos anos é resultado de muitas inadequações. Estão relacionadas ao controle inadequado dos vetores e reservatórios, ao aumento do número de casos da doença em pacientes imunossuprimidos (ex: pessoas com AIDS), ao aumento da resistência do agente ao tratamento e ao impacto causado pelas alterações climáticas globais, que refletem no aumento da transmissão de diversas outras doenças vetoriais (CALVOPINA; ARMIJOS; HASHGUCHI, 2004).

É perceptível que a prevalência da doença está centralizada em países com baixo poder econômico, ou seja, a maior parte da população afetada são pessoas em situação de pobreza, com baixa disposição de saneamento básico e políticas de saúde pública.

A cidade de Teófilo Otoni, localizada na região nordeste do estado de Minas gerais, conta com uma população de aproximadamente 141.934 mil habitantes segundo dados do censo de 2010. Sua uma área territorial é de $3.242,270 \mathrm{Km}^{2}$ e a vegetação prevalente é o cerrado com ocorrência de mata atlântica e predominantemente montanhosa (IBGE, 2018). O município se encontra no Vale do 
Mucuri, região de clima tropical semiúmido, com médias de temperaturas anuais de $23^{\circ} \mathrm{C}$.

A maior parte da população habita na área urbana da cidade. No censo de 2010 o IBGE registrou um IDH de 0,701(PNUD, 2011) porém é perceptível que em alguns bairros ainda há deficiência no sistema de saneamento básico.

$\mathrm{Na}$ cidade o setor responsável pelo controle de doenças endêmicas é a Vigilância Epidemiológica e Sanitária da Secretaria de Saúde. Esse órgão é responsável por buscar soluções de caráter preventivo que se encaixem no perfil socioeconômico do município para evitar doenças e agravos à saúde coletiva.

A modernização dos métodos de pesquisas vem trazendo resultados positivos no que diz respeito a conhecer os diversos fatores que agravam uma patologia. Os novos métodos de estudo foram utilizados ao longo dos anos em várias pesquisas com o intuito de direcionar e analisar a situação da saúde no país, auxiliando na elaboração de técnicas de controle e medidas que reduzam a propagação dos seus vetores.

Barcelos et al. (2008) citam que georreferenciamento de doenças é um método utilizado para mapear determinada área e realizar o processamento dos dados de registro de dada doeça. É uma ferramenta que permite indicar a localidade exata em uma posição geográfica. É utilizado atualmente como ferramenta de análise no sistema de saúde como forma de controle e prevenção de patologias. $\mathrm{O}$ mesmo permite a ampla visualização e distribuição espacial dos fatores de riscos presentes no ambiente.

Os mesmos autores definem o geoprocessamento como sendo "um conjunto de tecnologias voltadas para a coleta e tratamento de informações espaciais com determinado objetivo, executadas por sistemas específicos para cada aplicação". Sendo assim, a técnica de georreferenciar determinada doença pode ser facilmente aplicada por pesquisadores da área da saúde. Através do geoprocessamento de dados é possível conhecer o ambiente onde o indivíduo afetado está inserido, permitindo visualizar fatores que contribuem para o agravo. Neste caso é necessário atentar que a manifestação de uma doença pode estar relacionada a variáveis como condições sociais, demográficas, ambientais e econômicas.

O processamento dos dados de uma pesquisa pode ser realizado por programas de softwares do sistemas de informações geográficas (SIG) e hardware 
(GPS)que possibilitam a criação de mapas e gráficos dirigidos para análise do objeto de estudo.

O SIG vem se mostrando uma ferramenta de grande importância na pesquisa de adventos relacionados à saude. Em 2004 pesquisadores realizaram um estudo para localizar casos de dengue e a presença do seu vetor na cidade de Porto Alegre-RS, para atravez do geoprocessamento identificar características socioambientais que contribuiram para esse evento (BARCELOS et al., 2004).

Outro trabalho que utilizou o SIG, foi realizado no Hospital Universitário Clemente de Faria (HUCF), em Montes Claros e vizou destacar a distribuição espacial de paciente hospitalizados com Doenças Tropicais Negligenciadas na região norte de Minas Gerais (SILVA et al., 2016).

O vetor da LV possui características de reprodução que permitem fácil localização do seu foco e isso, consequentemente, possibilita a prática de medidas profiláticas específicas e direcionadas.

O presente estudo se baseia na premissa de o georreferenciamento de doenças ser utilizado como ferramenta fundamental a fim de garantir o controle na situação endêmica de LV no município de Teófilo Otoni-MG.O trabalho teve como objetivo analisar a incidência de casos confirmados de leishmaniose visceral nos diferentes bairros do município de Teófilo Otoni do ano de 2008 a 2017,identificando as áreas de maior risco de proliferação do mosquito transmissor da LV, caracterizar os bairros mais acometidos pela patologia e mensurando o risco de uma epidemia da doença na cidade, de modo a demonstrar a eficácia do georreferenciamento como ferramenta de planejamento de estratégias no controle da leishmaniose visceral.

\section{Metodologia}

O estudo se trata de uma pesquisa aplicada, de natureza quantitativa, em que foi realizado um levantamento de dados para explorar a distribuição espacial da leishmaniose visceral na cidade de Teófilo Otoni MG.

Os dados vetoriais da cidade e sua divisão em bairros foram obtidos gratuitamente através da plataforma do Instituto Brasileiro de Geografia e Estatística (IBGE) do censo de 2010. As características socioeconômicas e demográficas do 
município também foram extraídas da mesma plataforma disponível para consulta na internet.

Foi realizada uma busca documental de todas as notificações de LVem Teófilo Otoni no período de 2008 a 2017apartir da análise da base de dados do SINAN, bem como sua classificação quanto ao sexo e faixa etária. Após, esses dados foram confrontados com os dados cedidos pela Superintendência Regional de Saúde (SRS) afim de atestar sua veracidade. Os casos notificados registrados na plataforma do SINAN, estavam de acordo com os dados da SRS.

Os locais dos casos confirmados foram manipulados por meio de dispositivos computacionais dos Sistemas de Informações Geográficas QGis $\AA_{\text {, }}$ versão 2.14.16 para a elaboração dos mapas. As coordenadas dos casos foram obtidas por meio da plataforma Google Earth $\operatorname{Pro} \AA$ e armazenadas em forma tabelas utilizando as planilhas do programa Libre Office Calc®.

\section{Resultados e discussão}

A pesquisa contou o apoio da Superintendência Regional de Saúde de Teófilo Otoni, que disponibilizou os dados necessários para o progresso do estudo. O levantamento aponta que de 2008 a 2017 foram confirmados 19 casos de LV em residentes da cidade.

Dos 19 casos registrados foi observado que a maioria era do sexo masculino, como apresentado na TABELA1. A população masculina representou $79 \%$ dos casos confirmados, enquanto a feminina configura $21 \%$. Resultados semelhantes foram encontrados por Nogueira e Moreira durante um estudo que analisava as características epidemiológicas da LV no estado de Minas Gerais no período de 2007 a 2011 (NOGUEIRA; MOREIRA, 2013). Não há um estudo científico que justifique a grande diferença de infecção por sexo, porém pode estar relacionada com o quão expostos ao vetor os homens ficam em relação às mulheres por frequentarem constantemente regiões de matas e florestas(BORGES et al., 2008). No presente estudo, $37 \%$ dos casos confirmados compreende adultos em idade ativa para trabalho.

TABELA 1 -Morbidade da leishmaniose visceral por gênero em Teófilo Otoni-MG de2008 a 2017 


\begin{tabular}{cc}
\hline Gênero & no de casos \\
\hline MASCULINO & 15 \\
FEMININO & 4 \\
TOTAL & 19 \\
\hline
\end{tabular}

Fonte: Secretaria Municipal de Saúde de Teófilo Otoni/SINAN (2018)

Quanto à faixa etária foi percebido que a população infantil afetada foi superior à de adultos. 57,90\% dos casos notificados foram crianças com até 14 anos de idade, contra $42,1 \%$ de adultos e idosos no mesmo período. As populações adulto e senil, representaram cada uma $21,05 \%$ dos casos de leishmaniose visceral na cidade de Teófilo Otoni conforme TABELA 2.

Borges et. al.(2008) explicam que essa grande incidência na população infantil pode estar relacionada com o intenso contato que crianças tem com animais, além de em alguns casos apresentarem carência nutricional e debilidade do sistema imunológico que ainda está em desenvolvimento.

Entretanto um estudo realizado no Instituto Materno Infantil de Pernambuco (IMIP) no estado de Pernambuco constatou que o tempo de evolução da LV em 86\% das crianças era inferior a 60 dias e que era pouco provável que isso seja decorrente das alterações nutricionais crônicas que a doença causa (QUEIROZ; ALVES; CORREIA, 2004).

TABELA 2.Morbidade da leishmaniose visceral por faixa etária em Teófilo Otoni-MG de 2008 a 2017

\begin{tabular}{ccccccccc}
\hline Faixa Etária & 1 a 4 & $\begin{array}{c}5 \text { a } \\
9\end{array}$ & 10 a 14 & 20 a 39 & 40 a 59 & 70 a 79 & Total \\
\hline no de casos & 6 & 2 & 3 & 4 & 3 & 1 & 19 \\
\hline
\end{tabular}

Fonte: Secretaria Municipal de Saúde de Teófilo Otoni/SINAN (2018)

A TABELA 3 mostra a morbidade da LV por bairro de Teófilo Otoni. Dos 39 bairros catalogados, 12 possuem registro de notificação para a patologia. $O$ vetor da LV se reproduz em áreas com presença de material orgânico em decomposição. Os bairros onde foram registrados os maiores índices possuem características que expõe a população ao vetor. 
É perceptível que a maioria destes casos estão concentrados na região noroeste da cidade, áreas com menor disposição de saneamento básico, cercada por matas e com maiores possibilidades de focos do vetor da LV.

TABELA 3.Morbidade da leishmaniose visceral por bairro em Teófilo Otoni-MG De 2008 a 2017

\begin{tabular}{cc}
\hline $\begin{array}{c}\text { Bairros com casos } \\
\text { confirmados }\end{array}$ & $\begin{array}{c}\text { Número de casos } \\
\text { confirmados }\end{array}$ \\
\hline São Cristóvão & 4 \\
Bela Vista & 4 \\
Joaquim Pedrosa & 2 \\
Cidade Alta & 1 \\
Centro & 1 \\
Fátima & 1 \\
Frei Dimas & 1 \\
Jardim Iracema & 1 \\
Palmeiras & 1 \\
Altino Barbosa & 1 \\
São Jacinto & 1 \\
Teófilo Rocha & 1 \\
\hline
\end{tabular}

Fonte: Secretaria Municipal de Saúde de Teófilo Otoni/SINAN(2018)

O mapa temático(FIGURA 2) demonstra a intensidade de casos de LV nos bairros da cidade. Os bairros de maior ocorrência são o Bela Vista e São Cristóvão com $21,06 \%$ cada, seguidos por Joaquim Pedrosa que representa $10,52 \%$ dos casos confirmado. Os demais bairros com registro de casos apresentaram o mesmo percentual $(5,26 \%)$ de infecção por LV no período de estudo.

O localização espacial dos casos de LV está exposto na FIGURA 3, em que observa-se a posição geográfica exata dos casos registrados. Os pontos representam a localização com maior proximidade do local de ocorrência, permitindo 
concluir que o possível posicionamento geográfico do foco da doença pode estar onde há maior aglomeração de indivíduos notificados.

Na FIGURA 3 é possível visualizar que em 10 anos de ocorrência, houve uma centralização da ocorrência da doença na mesma localidade.

FIGURA 2. Mapa temático com apresentação da morbidade de leishmaniose visceral na cidade de Teófilo Otoni-MG de 2008 a 2017

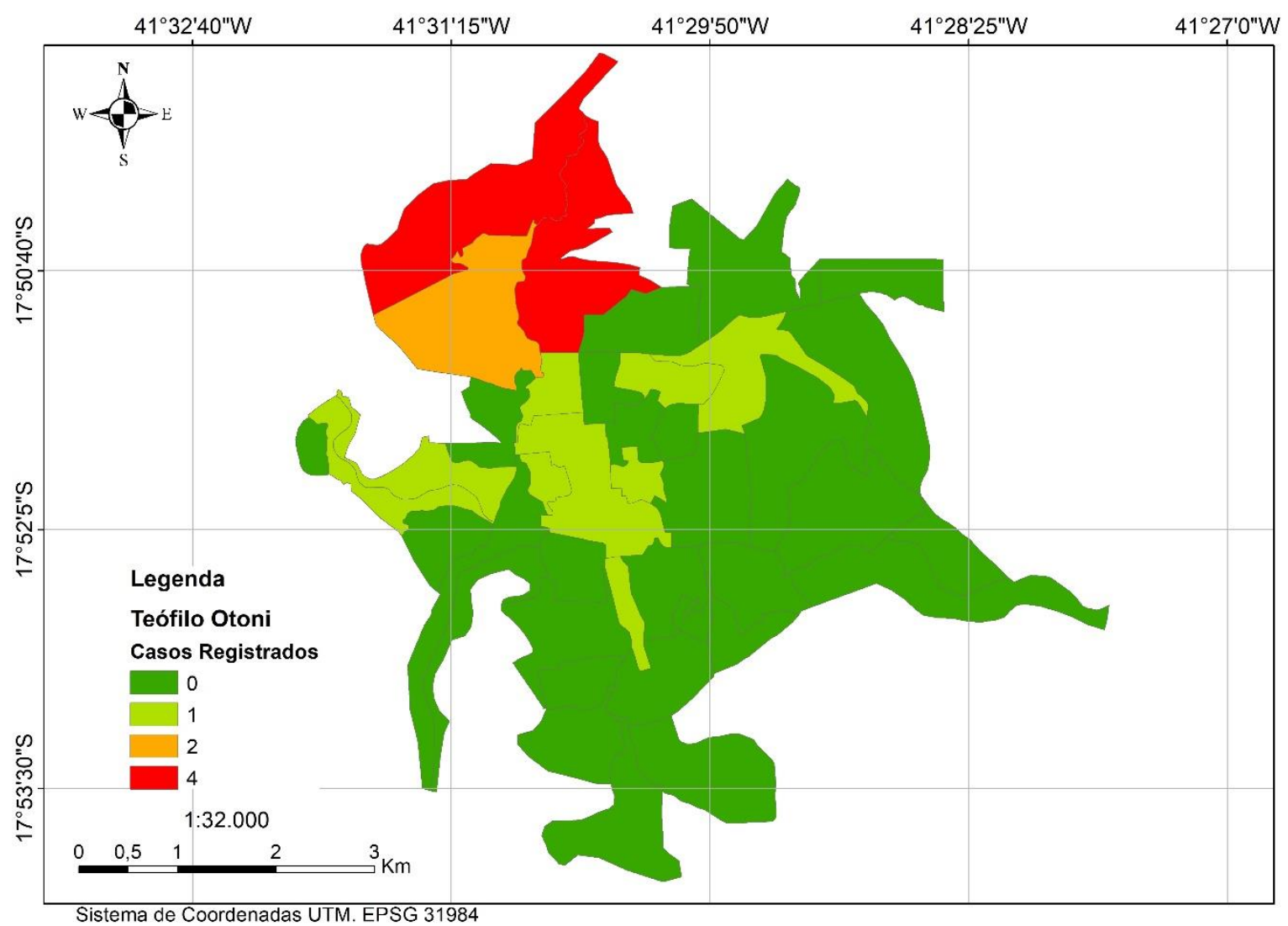

Fonte: Dados da própria pesquisa

Durante a pesquisa houve uma dificuldade em obter os dados geográficos atualizados da cidade, pois o arquivo fornecido pela Prefeitura Municipal apresenta falhas no cadastramento dos bairros, sendo que alguns são inexistentes no documento.

Entretanto essa falha não interferiu nos resultados da pesquisa, visto que 0 posicionamento geográfico é obtido com base nos endereços dos pacientes e as ruas apresentavam-se cadastradas em bairros vizinhos. 
As condições socioeconômicas e ambientais refletem diretamente no aumento dessa patologia em uma localidade. O georreferenciamento permite compreender o espaço em estudo e identificar as falhas, propiciando a elaboração de medidas preventivas contra a doença.

FIGURA 3. Georreferenciamento dos casos de leishmaniose visceral na cidade de Teófilo Otoni-MG no ano de 2008 a 2017

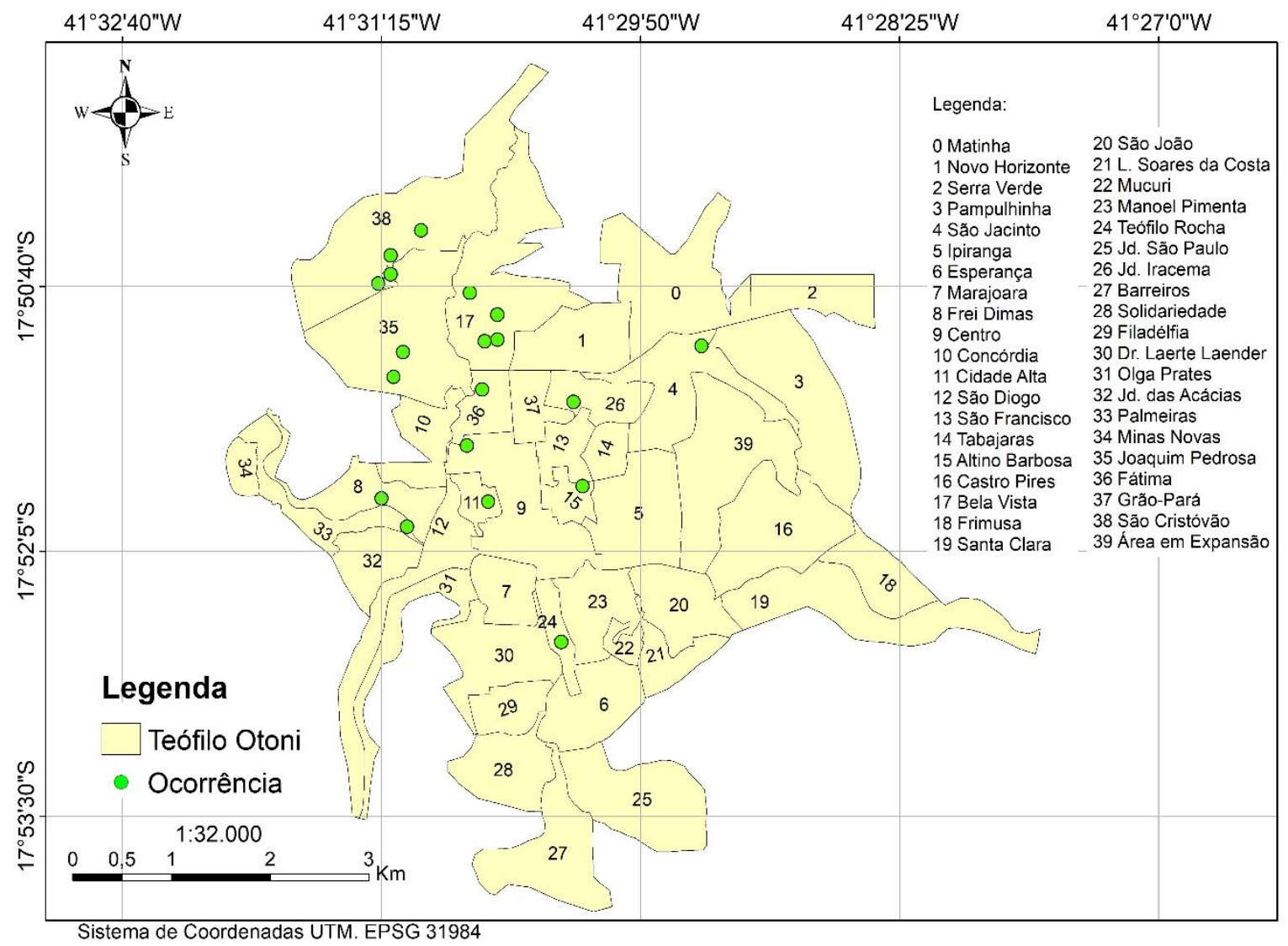

Fonte: Dados da própria pesquisa

Vê-se que mesmo com a programação regular na coleta de lixo, muitas ruas e terrenos vazios apresentam acúmulo por um período considerável. Outro agravo percebido é que por se tratar de uma região periférica abundantemente cercada por mata, é comum a presença de galinheiros, chiqueiros e pomares próximos ou no mesmo quintal das residências.

Destaca-se inclusive a grande população canina na cidade, principalmente os cães de rua que não recebem cuidados de prevenção adequados e se tornam 
mais vulneráveis ao contágio da doença. Considerados os principais reservatórios da LV, os cães devem ser sacrificados após a confirmação da doença uma vez que ainda não há um tratamento eficaz.

Essas condições podem explicar o fato de os casos de LV nesta localidade estarem tão próximos um do outro.

A população tem papel imprescindível no controle da LV na cidade, tanto nos cuidados com seus animais domésticos e prevenção da proliferação do vetor, quanto na exigência junto à prefeitura municipal da aplicabilidade adequada dos recursos de saúde às regiões de maior necessidade.

A LV é considerada uma doença tropical negligenciada (DTN), assim como chagas, esquistossomose, tuberculose, malária, entre outras. Essas doenças apresentam grandes índices de mortalidade nos países mais afetados e despertam pouco interesse dos governantes e da indústria farmacêutica no desenvolvimento de medidas de combate e na pesquisa de novos medicamentos.

Percebe se que as ações de combate à LV e outras doenças infecto contagiosas no país são demasiadamente inferiores às ações de combate a doenças como dengue, HIV e febre amarela. Isso se dá pelo impacto imediato que essas doenças causam, principalmente por atingir classes sociais mais altas da população, enquanto as doenças negligenciadas estão ligadas às condições de pobreza.

Neste caso, é possível entender que os investimentos são mais concentrados em ações de combate a doenças que movimentam a economia e geram marketing para seus idealizadores.

As iniciativas privadas apresentam maior interesse em desenvolver pesquisas de controle e erradicação das doenças negligenciadas. Um exemplo é a Iniciativa de Medicamentos para Doenças Negligenciadas (DNDi, em inglês), uma organização sem fins lucrativos que objetiva desenvolver pesquisas voltadas à criação de medicamentos para as DTN's. No Brasil, essa organização conta com o apoio da Fiocruz (BRASIL, 2006).

Esses estudos desenvolvidos são de grande valia para os gestores da saúde, e com a ferramenta de georreferenciamento há a possibilidade de modelar os programas desempenhados pelas políticas públicas de saúde, para que os diversos fatores que agravam uma patologia, sejam primeiramente conhecidos, e 
posteriormente sejam desenvolvidas medidas eficazes, que intensifiquem a atenção ao tratamento medicamentoso, diminuam os focos de proliferação dos vetores existentes na cidade e desenvolva praticas junto à população a fim de expor o real quadro endêmico da leishmaniose na cidade de Teófilo Otoni-MG. A prática de medidas preventivas eficientes é imprescindível para a erradicação da leishmaniose visceral.

\section{Conclusão}

A leishmaniose visceral está difundida em grande parte de Teófilo Otoni, com maior concentração na região noroeste. A sua disposição espacial permitiu visualizar as áreas de maior risco que necessitam de maior assistência de políticas públicas voltadas à prevenção da patologia. Foi percebido que os aspectos socioeconômicos e ambientais são significativos na centralização dos casos de LV na cidade.

O georreferenciamento de doenças tem se mostrado eficaz no que diz respeito ao monitoramento de áreas com grande morbidade, mortalidade $\mathrm{e}$ incidência de doenças infecto parasitárias, portanto sua aplicabilidade deve ser constante. $O$ geoprocessamento de dados de saúde pode ser considerado uma ferramenta fundamental que contribui para a organização, planejamento e tomada de decisões.

Segundo dados da GRS foi percebido um aumento dos casos a partir de 2014, o que tornou a região endêmica para a leishmaniose. Logo, no contexto do estudo, as medidas de combate ao vetor devem ser direcionadas às áreas de risco identificadas, para melhores resultados no controle da leishmaniose visceral.

\section{Referências}

ALVARENGA, D.G.et al.Leishmaniose visceral: estudo retrospectivo de fatores associados a letalidade. Rev da Soc Bras de Med Trop.v. 43, n.2,p. 194-197. 2010. Disponível em: <http://www.scielo.br/scielo.php?pid=S003786822010000200017\&script=sci_abstract\&tlng=pt>. Acesso em: 14 mar. 2018.

BARATA, R.A. et al. Controle da leishmaniose visceral no município de Porteirinha, Estado de Minas Gerais, no período de 1998 a 2003. Rev da Soc Bras de Med Trop. v.44, n.3, p. 386-388, 2011. Disponível em: $<$ www.scielo.br/pdf/rsbmt/v44n3/v44n3a25.pdf>. Acesso em: 04 abr. 2018. 
BARCELLOS, C. et al. Georreferenciamento de dados de saúde na escala submunicipal: algumas experiências no Brasil. Epidemiol. Serv. Saúde. Brasília, v. 17, n. 1, p. 59-70, 2008. Disponível em:

<https://www.arca.fiocruz.br/handle/icict/1290>. Acesso em: 27 abr. 2018.

BARCELLOS, C. et al. Identificação de locais com potencial de transmissão de dengue em Porto Alegre através de técnicas de geoprocessamento. Rev. Soc. Bras. Med. Trop. v. 38, n.3, p. 246-250, 2004. Disponível em:

$<$ http://www.scielo.br/scielo.php?script=sci_arttext\&pid=S0037-86822005000300008. Acesso em: 10 maio 2018.

BORGES, B.K.A. et al. Avaliação do nível de conhecimento e de atitudes preventivas da população sobre a leishmaniose visceral em Belo Horizonte,Minas Gerais, Brasil. Cad. Saúde Púb. v. 24, n. 4, p. 777-784. 2008. Disponível em: <http://www.scielo.br/scielo.php?pid=S0102-

311X2008000400007\&script=sci_abstract\&tlng=pt>. Acesso em: 16 maio 2018.

BRASIL. Ministério da Saúde.Manual de vigilância e controle da leishmaniose visceral. Brasília: Secretaria de Vigilância em Saúde. Departamento de Vigilância Epidemiológica, 2006. Disponível em:

$<$ http://bvsms.saude.gov.br/bvs/publicacoes/manual_vigilancia_controle_leishmanios e_visceral.pdf>. Acesso em: 30 mar. 2018]

CALVOPINA, M.; ARMIJOS, R.X.; HASHGUCHI; Y. Epidemiology of leishmaniasis in Ecuador: current status of knowledge: a review. Memórias do Instituto Oswaldo Cruz, Rio de Janeiro, v. 99, n. 7, p. 663-672, 2004. Disponível em: $<$ http://www.scielo.br/scielo.php?script=sci_arttext\&pid=S007402762004000700001 >. Acesso em: 15 abr. 2018.

IBGE - Instituto Brasileiro de Geografia e Estatística.Brasil em síntese. Disponível em: <https://cidades.ibge.gov.br/brasil/mg/teofilo-otoni/panorama>. Acesso em: 22 abr. 2018.

MICHALICK, M.S.M.; GENARO, O. Leishmaniose visceral americana. In: NEVES, D.P. et al.Parasitologia humana. 11. ed. São Paulo: Editora Atheneu, 2005. p. 67-83.

MINAS GERAIS. Secretaria de Saúde do Estado de Minas Gerais. Boletim Epidemiológico - Leishmaniose Visceral Humana 2010-2015. Belo Horizonte, 2017 Disponível em: <http://vigilancia.saude.mg.gov.br/index.php/download/boletimepidemiologico-leishmaniose-visceral-humana-minas-gerais-2010-2015/>. Acesso em: 08 abr. 2018.

MURRAY, H.W. Treatment of visceral leishmaniasis in 2004.Am. J. Trop. Med. Hyg. v. 71, n. 6. p. 787-794, 2004. Disponível em: <https://pdfs.semanticscholar.org/440a/67a2859017e722693377c3050aa0e8cf9bc9.p df?.ga $=2.214883543 .175079254 .1526958494-976903944.1526958494>$. Acesso em: 21 mar. 2018. 
NOGUEIRA, G.A.; MOREIRA,S.M. Características epidemiológicas associadas à ocorrência de leishmaniose no estado de Minas Gerais, Brasil (2007-2011), 2013. Disponível em: http://www3.izabelahendrix.edu.br/ojs/index.php/aic/article/view/404>. Acesso em: 15 maio 2018.

OLIVEIRA, E.N. Perfil Epidemiológico da leishmaniose visceral no município de Paracatu-MG, no período de 2007 a 2010. 2011. 54 p. Monografia (Graduação em Ciências Biológicas) - Uberaba. Universidade Federal de Minas Gerais, 2011 Disponível em: <https://www.nescon.medicina.ufmg.br/biblioteca/imagem/3103.pdf>. Acesso em: 21 mar. 2018.

PNUD - Programa das Nações Unidas para o Desenvolvimento. Ranking decrescente do IDH-M dos municípios do Brasil. Atlas do Desenvolvimento Humano, 2010.

Disponível em: <http://www.atlasbrasil.org.br/2013/pt/ranking>. Acesso em: 22 abr. 2018.

QUEIROZ, M.J.A.; ALVES, J.G.B.; CORREIA, J.B. Leishmaniose visceral: características clínico-epidemiológicas em crianças de área endêmica. J. Pediatr. (Rio J.). 2004. v.80, n. 2,p. 141-146. Disponível em: http://www.scielo.br/scielo.php?script=sci_arttext\&pid=S002175572004000200012\&lng=en. http://dx.doi.org/10.1590/S002175572004000200012. Acesso em: 18 maio 2018.

SILVA, D.V.A. et al. Georreferenciamento dos Casos de Doenças Tropicais Negligenciadas em Municípios do Norte de Minas Gerais. Fórum de Ensino Pesquisa e Extensão da Universidade Estadual de Montes Claros, 2016. Disponível em: <https://www.fepeg2016.unimontes.br/index.php/anais/ver/211. Acesso em: 14 mai 2018.

WHO - World Health Organization. Annual report Control of the leishmaniases. Expert Committee, Geneva - Switzerland, 1990. Disponível em:

$<$ http://apps.who.int/iris/bitstream/handle/10665/44412/WHO_TRS_949_eng.pdf?seq uence=1>. Acesso em: 28 mar. 2018. 